\title{
Long-Term Memory Leads to Synaptic Reorganization in the Mushroom Bodies: A Memory Trace in the Insect Brain?
}

\author{
Benoît Hourcade, ${ }^{1}$ Thomas S. Muenz, ${ }^{2}$ Jean-Christophe Sandoz, ${ }^{1}$ Wolfgang Rössler, ${ }^{2}$ and Jean-Marc Devaud ${ }^{1}$ \\ ${ }^{1}$ Research Centre on Animal Cognition, Centre National de la Recherche Scientifique-Université Paul-Sabatier, Unité Mixte de Recherche 5169, 31062 \\ Toulouse cedex 04, France, and 2Department of Behavioral Physiology and Sociobiology, Biozentrum, University of Würzburg, D-97074 Würzburg, Germany
}

The insect mushroom bodies (MBs) are paired brain centers which, like the mammalian hippocampus, have a prominent function in learning and memory. Despite convergent evidence for their crucial role in the formation and storage of associative memories, little is known about the mechanisms underlying such storage. In mammals and other species, the consolidation of stable memories is accompanied by structural plasticity involving variations in synapse number and/or size. Here, we address the question of whether the formation of olfactory long-term memory (LTM) could be associated with changes in the synaptic architecture of the MB networks. For this, we took advantage of the modular architecture of the honeybee MB neuropil, where synaptic contacts between olfactory input and MB neurons are segregated into discrete units (microglomeruli) which can be easily visualized and counted. We show that the density in microglomeruli increases as a specific olfactory LTM is formed, while the volume of the neuropil remains constant. Such variation is reproducible and is clearly correlated with memory consolidation, as it requires gene transcription. Thus stable structural synaptic rearrangements, including the growth of new synapses, seem to be a common property of insect and mammalian brain networks involved in the storage of stable memory traces.

\section{Introduction}

In insects, especially in fruitflies and honeybees, the mushroom bodies (MBs) have been assigned an important role in the formation of olfactory associative memories (Davis, 2005; Giurfa, 2007). In a recent study on Drosophila, Krashes et al. (2007) elegantly showed that different subsets of MB neurons (the Kenyon cells) interact in a dynamic fashion to form and stabilize associative olfactory memories. In honeybees, the MBs are also involved in the formation, consolidation and recall of olfactory memories (Erber et al., 1980; Locatelli et al., 2005; Devaud et al., 2007). However, the neural bases of long-term storage in these brain structures remain unknown. Are MB neurons structurally modified when long-term memory (LTM) is consolidated? The MBs are highly plastic structures which undergo profound changes with the insect's experience, especially foraging (Withers et al., 1993; Durst et al., 1994; Farris et al., 2001; Groh et al., 2004, Krofczik et al., 2008). However, to our knowledge the contribution of memory storage per se to stable structural changes in higher-order brain centers has never been evaluated in insects. Here, we demonstrate robust changes in the synaptic architecture

\footnotetext{
Received Feb. 16, 2010; revised March 22, 2010; accepted March 28, 2010.

This work received financial support from the Centre National de la Recherche Scientifique and Université PaulSabatier to J.C.S. and J.M.D., and from a Deutsche Forschungsgemeinschaft grant (SFB554-A8) to W.R. We thank Sandrine Paute for beekeeping and the collection of bees at emergence. The SYNORPH1 anti-synapsin I antibody developed by Erich Buchner was obtained from the Developmental Studies Hybridoma Bank developed under the auspices of the National Institute of Child Health and Human Development and maintained by The University of lowa, Department of Biological Sciences, lowa City, IA.

Correspondence should be addressed to Jean-Marc Devaud, at the above address. E-mail: devaud@cict.fr.

J.C. Sandoz's present address: Evolution, Genomes and Speciation Laboratory, CNRS (UPR 9034), 91198 Gif sur Yvette, France.

DOI:10.1523/JNEUROSCI.0841-10.2010

Copyright $\odot 2010$ the authors $\quad 0270-6474 / 10 / 306461-05 \$ 15.00 / 0$
}

of the MB input region (calyces) following the consolidation of a stable, transcription-dependent, memory of a specific odorant.

\section{Materials and Methods}

Animals. Honeybees (Apis mellifera ligustica) were collected at emergence and kept in rearing boxes for $7 \mathrm{~d}$. They were then cooled and restrained in harnesses allowing free movement of antennae and mouthparts (Bitterman et al., 1983), fed with $4 \mu \mathrm{l}$ of $50 \% \mathrm{w} / \mathrm{w}$ sucrose solution and maintained for $3 \mathrm{~h}$ in a dark chamber $\left(25^{\circ} \mathrm{C}, \sim 75 \%\right.$ humidity $)$

Behavior. Learning consisted of an associative conditioning of the proboscis extension reflex (PER), in which the bees form an association between an odorant [the conditioned stimulus (CS)] and a sucrose reward [unconditioned stimulus (US)] (Bitterman et al., 1983). In paired groups, bees received 5 trials with an intertrial interval of $10 \mathrm{~min}$. At each trial, the CS (1-nonanol; 98\%, Sigma-Aldrich) was presented for 4 s and then the US (50\% w/w sucrose solution) for $3 \mathrm{~s}$, with an interstimulus interval of $3 \mathrm{~s}$ ( $1 \mathrm{~s}$ overlap). In the naive group (first control), bees were placed 5 times in the setup without any stimulation to equalize experience with the experimental context. In the unpaired group (second control), bees received explicitly unpaired presentations of the CS and the US (5 odor-only and 5 sucrose-only presentations, $5 \mathrm{~min}$ apart in a pseudorandomized sequence). As a third control, bees from the paired ActD group received the same conditioning procedure as in the paired group, but were injected with $1 \mu \mathrm{l}$ of $1.5 \mathrm{~mm}$ Actinomycin D (Sigma-Aldrich) using a precision syringe (Hamilton) into their thorax, $3 \mathrm{~h}$ after learning. We previously showed that these conditions allowed to abolish LTM (Hourcade et al., 2009). To control for possible effects of the injection on memory, bees from all the other groups were injected with $1 \mu$ l of PBS following the same procedure. All animals were fed twice a day with $4 \mu \mathrm{l}$ of $50 \% \mathrm{w} / \mathrm{w}$ sucrose solution and were kept in a dark chamber $\left(25^{\circ} \mathrm{C}\right.$, $\sim 75 \%$ humidity) until retrieval tests were conducted, $3 \mathrm{~d}$ after training.

To assess LTM, bees were given two unrewarded odor presentations, of 1-nonanol (the CS, or learned odor) and of 1-hexanol (a novel odor) $(>99 \%$, Fluka), in alternated order across individuals. The percentage of 
specific responses to the learned odor was calculated by subtracting the percentage of responses induced by the novel odor from that of responses evoked by the CS. After the odor tests, bees showing no response to sucrose $(<5 \%)$ were discarded.

Immediately after the retrieval tests, paired bees showing a specific response to the CS and control bees responding to neither of the two odors were killed and dissected. The proportions of bees displaying such response patterns used for selection in the corresponding groups did not differ significantly $\left(\chi^{2}=6.22, \mathrm{df}=3, p>0.05\right)$ (Naive: $87.1 \%$; Paired: 57.7\%; Paired ActD: 76.3\%; Unpaired: $86.1 \%$ ). Thus, in all groups the bees taken for analysis were representative, and any anatomical difference between groups would not reflect a biased selection procedure. The brains were dissected in cold PBS, immersed overnight in 4\% paraformaldehyde in $0.1 \mathrm{M} \mathrm{PBS}$ at $4^{\circ} \mathrm{C}$, washed 3 times and processed as follows. Before image analysis, all confocal files were encoded so that the whole procedure [i.e., selection of the circle locations, counting of microglomeruli, contour tracing, and three-dimensional (3D) reconstruction of the lips] was conducted blindfold with respect to treatment.

Microglomeruli double staining and density measurements. Microglomeruli were labeled and quantified adapting a published protocol for double staining presynaptic and postsynaptic elements (Groh et al., 2004; 2006; Krofczik et al., 2008). Brains were embedded in 5\% low meltingpoint agarose (Agarose II, no. 210-815, AMRESCO) and sectioned in a frontal plane $(100 \mu \mathrm{m})$ with a vibrating microtome (Leica VT 1000S). Free-floating sections were preincubated in PBS with $0.2 \%$ Triton X-100 and $2 \%$ normal goat serum, then incubated simultaneously in $0.2 \mathrm{U}$ of Alexa Fluor 488 phalloidin (Invitrogen, A-12379) and with a monoclonal anti-synapsin I antibody (1:50; SYNORF1; Developmental Studies Hybridoma Bank, University of Iowa, Iowa City, IA). Double-labeled preparations were incubated in the secondary antibody (Alexa Fluor 546-conjugated goat anti-mouse, Invitrogen: 1:250 in PBS with 1\% normal goat serum) for $2 \mathrm{~h}$ at room temperature. Central sections containing the central body, the peduncles and the MB lips were scanned with a laser-scanning confocal microscope (TCS5, Leica), with $63 \times$ oilimmersion objective. Excitation and emission wavelengths were respectively $561 \mathrm{~nm} / 570-620 \mathrm{~nm}$ (Alexa 546) and $488 \mathrm{~nm} / 500-550 \mathrm{~nm}$ (Alexa 488). Within each brain two optical sections containing the medial lips and collars of the two medial calyces were taken $15 \mu \mathrm{m}$ apart. The two channels were merged using pseudocolors in ImageJ software (Wayne Rasband, National Institutes of Health, Bethesda, MD). On each optical section, two circles of $400 \mu \mathrm{m}^{2}$ (in rostral and caudal positions) were drawn within each lip and collar (8 circles in total for each of these neuropils per animal). Microglomeruli, as identified by a clear presynaptic and postsynaptic staining on the merged image, were counted individually in each circle. This allowed calculation of the mean microglomerular density per circle, averaged over 8 circles (no significant differences were found between rostral and caudal areas in the same lip, between brain hemispheres, or between different optical sections).

Staining procedure for volume analyses of the MB lips. Brains were stained $3 \mathrm{~h}$ in neutral red (4\% in PBS, Michrome ${ }^{\circ}{ }^{2} 26$, Edward Gurr, Ltd.), then rinsed, dehydrated, and clarified in methyl salicylate (SigmaAldrich) for at least $4 \mathrm{~d}\left(\right.$ at $\left.-20^{\circ} \mathrm{C}\right)$ until observation. Whole-mounts were visualized under the confocal microscope with a $20 \times$ waterimmersion objective. Preparations were excited at $561 \mathrm{~nm}$ and the fluorescence emission was detected at $575-650 \mathrm{~nm}$. Within each bee, frontal optical sections $(512 \times 512$ pixels $)$ were taken at $5 \mu \mathrm{m}$-intervals in the region of $\mathrm{MB}$ calyces. Complete stacks were then imported in Amira 3.1 (Mercury Computer Systems). The medial lip of each medial calyx (where the microglomerular density had been estimated) and central body were reconstructed by tracing manually their contours on each section (the medial axis of the calyx was taken as the lateral limit). The software provided a volume estimate of each reconstructed structure by extrapolation from the drawn serial surfaces (see Fig. $3 A$ ). The mean volume was then calculated as the average of two measurements per animal.

Statistical analysis. Acquisition performances along trials were assessed within each group using Cochran's $Q$ test. Overall performance (the sum of responses during conditioning) was compared between groups using Mann-Whitney $U$ test. Retention performances at $3 \mathrm{~d}$ were analyzed
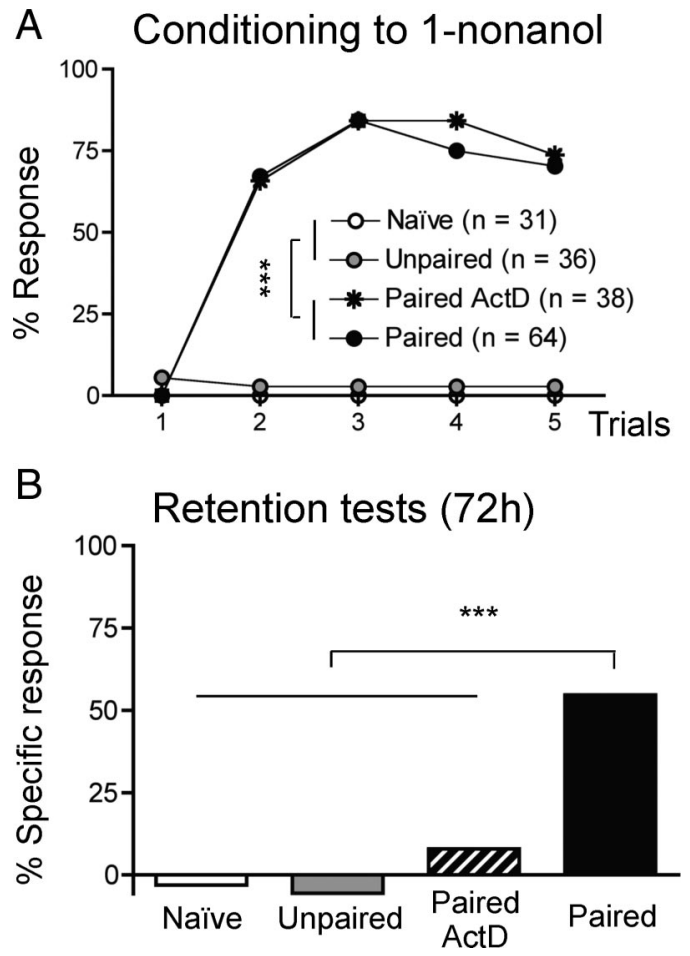

Figure 1. Spaced olfactory conditioning leads to transcription-dependent, odorant-specific, late long-term memory. $A$, Learning performances are measured as the percentage of conditioned responses (PER elicited by the CS). Over the 5 trials of conditioning, only bees from the paired groups (including those later treated with Actinomycin D: paired ActD group) formed the CS-US association. Almost no conditioned responses were produced when the CS and the US were temporally dissociated (unpaired) or absent (naive). $\boldsymbol{B}$, Specific retention levels of the association assessed $72 \mathrm{~h}$ after conditioning, as the difference between responses to the learned odor (1-nonanol) and to a novel odor (1-hexanol). Bees from the paired group displayed significant levels of specific memory. Bees in which transcription was blocked after learning (paired ActD group) or those that had not learned (unpaired and naive groups) showed levels of specific responses not significantly different from $0 .{ }^{* *} p<0.001$.

between groups using $\chi^{2}$ tests. Mean microglomerular densities were compared across groups using a two-way ANOVA, with treatment (naive, unpaired, paired ActD vs paired) and calyx subdivision (lip vs collar) as factors. Volumetric data were subjected to a two-way ANOVA, with treatment (naive, unpaired, paired ActD vs paired) and brain structure (lip vs central body) as factors. All statistical tests were done using STATISTICA 5.0 (StatSoft).

\section{Results}

We performed a parallel assessment of putative synaptic density and neuropil volume in the MB input region, the calyx, in relationship with LTM. To evaluate changes specific for LTM, we compared brains of bees which formed an odorant-specific LTM (paired group) with those of bees without such LTM (naive, unpaired, and paired ActD groups).

\section{Paired bees develop an odorant-specific and transcription-dependent LTM}

In the appetitive Pavlovian task of PER conditioning, a conditioning protocol based on 5 spaced paired CS-US presentations leads to the formation of a robust LTM that is classically measured 3-4 d after conditioning in laboratory conditions, and can be abolished using transcription inhibitors like Actinomycin D: the late long-term memory (1-LTM), as opposed to the early form (e-LTM), requiring translation but not transcription (Schwärzel and Müller, 2006). Bees which received paired CS-US presenta- 


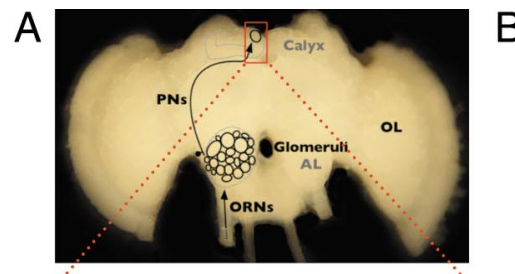

B
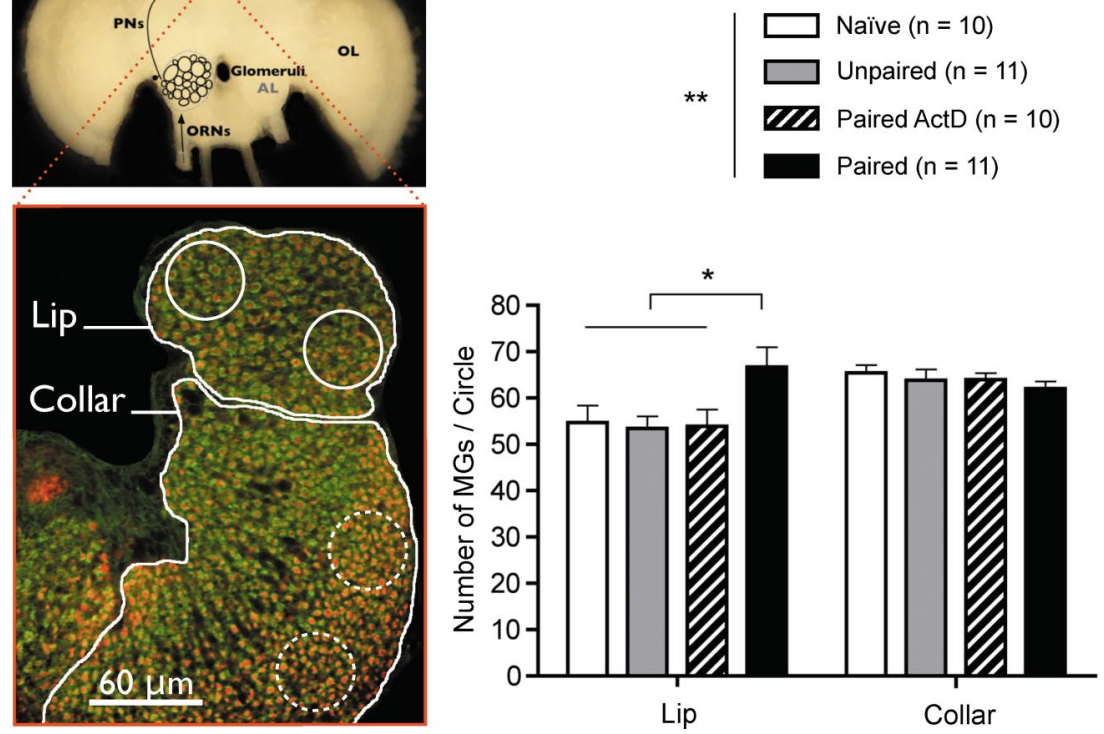

Figure 2. Microglomerular density is increased in the mushroom body lips when an olfactory memory is formed. $\boldsymbol{A}$, Microglomerular density was measured in each medial calyx (red frame), both in the lip — receiving olfactory input from the PNs exiting the $\mathrm{AL}$ —and in the collar—receiving visual input from the optic lobe (OL). ORNs, Olfactory receptor neurons. Microglomeruli were counted in $400 \mu \mathrm{m}^{2}$ circles placed in the medial lip (continuous outlines) and collar (dotted outlines) (see Materials and Methods). $B$, Estimated densities of microglomeruli averaged over 8 circles for both the lip and collar of each animal. Bees from the paired group showed a density significantly higher than that in the 3 control groups, in the lip only. ${ }^{*} p<0.05 ;{ }^{* *} p<0.01$.

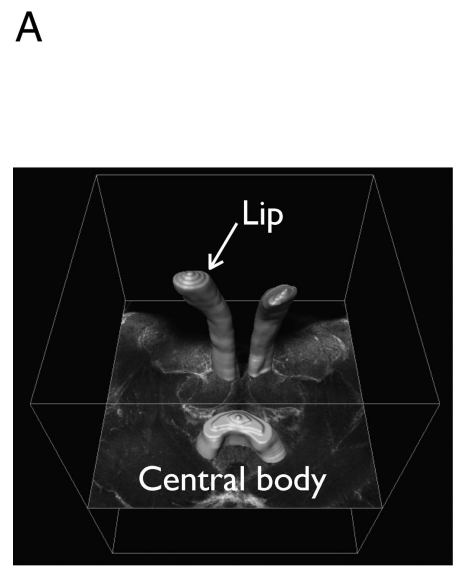

B

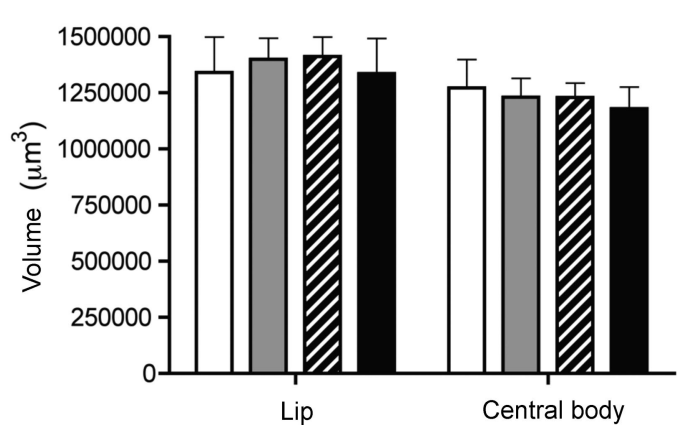

Figure 3. The lip volume is not affected by associative olfactory long-term memory. $\boldsymbol{A}$, The medial lips of the medial calyces (where microglomerular density was measured) were 3D-reconstructed and their volume estimated, as was the central body (CB), a nonolfactory center. $\boldsymbol{B}$, No differences were found between the mean volumes of the lip and central body of the different groups of individuals. NS, Nonsignificant.

tions (trials) showed an increase of conditioned responses to the CS (Fig. $1 A$, paired and paired-ActD, Cochran's $Q$ test, $Q>94.6$, $4 \mathrm{df}, p<0.001)$. As expected the temporal dissociation between the odorant and sucrose presentations yielded almost no learning (unpaired), nor did the experience of the setup only (naive). Three days later, only the paired group treated with PBS showed significant retention (McNemar's test: $\chi^{2}=33.03, p<0.001$ ) (Fig. $1 B$ ). ActD treatment prevented stable retention, as the response percentages of bees from the paired ActD group did not differ from zero $\left(\chi^{2}=1.33, p>0.05\right)$, like those of bees that had not learned initially (unpaired, naive; $\chi^{2}<0.5, p>0.05$ ). We verified that the proboscis extensions observed during the reten- tion test in the paired group were specifically induced by presentation of the CS (1-nonanol) and not of another odorant (1-hexanol) for most individuals (only 25\% responded to 1-hexanol). By contrast, most of the few control bees responding to the CS (8.3-23.7\%) also responded to the novel odorant (12.5-15.8\%), yielding levels of specific responses close to zero (Fig. $1 B$ ). Thus, in contrast to the controls, most individuals from the paired group had formed a stable and CS-specific memory $3 \mathrm{~d}$ after learning, and this memory was bona fide l-LTM requiring gene transcription.

\section{The synaptic architecture of the} olfactory MB neuropil is modified as LTM is formed

We hypothesized that, if CS-specific memory is related to changes in the synaptic architecture of the MB calyces, the number and/or density of synaptic complexes might differ between paired bees and bees from the three other groups, since only the former displayed an odorspecific memory. We visualized presynaptic and postsynaptic elements of microglomeruli (see Materials and Methods) and estimated their density in the lip and collar of the MB calyx (Fig. 2A). As a result (Fig. $2 B$ ), we found that, on average, the bees that had retained the olfactory memory for $3 \mathrm{~d}$ (paired) showed a higher density of microglomeruli (MGs) than those that had not formed such a memory (naive and unpaired) or which were experimentally prevented from consolidating the information after acquisition (paired ActD) (one-way ANOVA, $F_{(3,38)}=3.26, p<$ 0.05 ; post hoc $t$ tests, $t>2.62, p<0.05$ for all comparisons with the paired group). This was observed in the lip but not in the collar (respectively, olfactory and visual areas) (Gronenberg, 2001) (one-way ANOVA, $\left.F_{(3,38)}=0.59, p>0.05\right)$. Thus, the change in density in the lip was specifically related to the transcription-dependent storage of olfactory information in bees from the paired group, rather than to a nonspecific plastic process. This change was found independently in another experiment comparing untreated paired and unpaired bees of unknown age (see supplemental Material, available at www.jneurosci.org). This indicates that olfactory l-LTM is accompanied by a significant and reproducible increase in MG density that seems restricted to the olfactory network related with the storage of a CS-specific memory.

\section{The neuropil volume of the calyx-lip remains constant as LTM is formed}

We asked whether the MB lips might also display variations in size accompanying the variations in their synaptic organization. In fact, the observed increase in the MG density could be the mere consequence of a reduction in lip volume without any changes in 
the number of synaptic units. To quantify possible changes in volume, the MB lips from brains stained with neutral red were 3D-reconstructed (Fig. 3A) and their volumes estimated from serial optical sections (see Materials and Methods). The same was done for the central body, a midline brain neuropil involved in locomotion control, which was used as an internal reference. The results show no significant variation in the average volume of the lip (or central body) among the four groups of animals (Fig. $3 B$ ) (ANOVA, treatment $\times$ brain region, $F_{(3,31)}=0.17, p>0.05$ ). We therefore conclude that the induction of l-LTM resulted in an increase in MG density without any change in the total volume of the lip.

\section{Discussion}

This study shows that the formation of a transcriptiondependent LTM is accompanied by a specific, stable, increase in the density of microglomeruli in the insect MB. Changes in the microglomerular organization of $\mathrm{MB}$ lips were observed as a function of rearing temperature (Groh et al., 2004), age (Groh et al., 2006; Krofczik et al., 2008) or behavioral experience (Krofczik et al., 2008). However, we show for the first time that microglomerular organization can be modified by the formation of an odor-reward memory. Plasticity was modality-specific, as only olfactory (lips) but not visual areas (collars) were affected. Such a plasticity cannot be simply explained by differences in sensory experience per se. Indeed, MG density differed between animals sharing the same olfactory experience (paired and unpaired) while it was not affected by variations in olfactory input (the unpaired and naive groups show similar MG density values). Rather, the effect was only observed after associative olfactory learning followed by a transcription-dependent consolidation. Transcription-independent memories, such as early-LTM (Schwärzel and Müller, 2006) obviously did not lead to any detectable changes $3 \mathrm{~d}$ after conditioning, as the unpaired and paired $\mathrm{ActD}$ groups displayed equivalent MG density. Hence, either MG density remains unaltered as such memories are formed, or it is altered only transiently. In any case, a stable increase in MG density appears to be specifically linked to the formation of transcription-dependent, late LTM.

Structural plasticity in the MB calyces is not manifested exclusively as variations of MG density. Total neuropil volume may also undergo changes with experience, especially at the onset of foraging (bees, Withers et al., 1993; Maleszka et al., 2009; ants, Gronenberg et al., 1996; wasp, O'Donnell et al., 2004). Foraging is however a complex behavior involving spatial navigation, multimodal sensory discrimination and learning (Winston, 1987). Our results suggest that the formation of stable olfactory memories occurs without changes in lip volume. This may simply reflect the fact that under experimental conditions the number and complexity of stored olfactory memories are well below those actually formed under natural foraging conditions although MB volume seems uncorrelated with learning performance (Maleszka et al., 2009). Alternatively, an increased neuropil volume may result from greater sensory load. The detection and discrimination of many flower compounds during foraging would induce high activity in the olfactory pathway, thereby triggering the growth of neuronal elements in the lips (Farris et al., 2001; Jones et al., 2009; Seid and Wehner, 2009).

The LTM-related increase in MG density also differs from the general process of $\mathrm{MB}$ maturation, which involves a decrease in the density of MGs while the lip volume slightly increases (Durst et al., 1994; Muenz et al., 2008). This is likely due to progressive growth of Kenyon cell dendritic branches with increasing age and sensory experience (Farris et al., 2001; Muenz et al., 2008). Consistently, density was significantly higher in our 7-d-old bees (Fig. 2) than in more mature bees collected as they exited the hive (which they typically start to do at 2-3 weeks of age) (supplemental Fig. S1, available at www.jneurosci.org as supplemental material), regardless of memory (Mann-Whitney $U$ test: $U=184, p<$ 0.001). Thus, long-term information storage, intense sensory processing and age appear to contribute differently to complex structural synaptic changes in the MBs in the course of an animal's lifetime.

Since total neuropil volume remained unchanged, our results suggests a net increase in the total number of MG. As such an increase requires pairing between CS and US, it may be interpreted as activity-dependent plasticity, possibly resulting from changes in the presynaptic input from projection neurons (PNs). Five spaced CS-US presentations lead to increased calcium activity in the lip in response to the rewarded odorant (Faber and Menzel, 2001) which may be due to increased incoming input from the antennal lobe (AL) after learning (Faber et al., 1999). Indeed, plasticity of PN responses was observed both in Drosophila (Yu et al., 2004) and Apis (Fernandez et al., 2009). Supporting this view, LTM formation induces structural reorganizations in $\mathrm{AL}$ glomeruli, which could result in increased activity from a subset of the PNs (Hourcade et al., 2009). Even so, the observed changes would be widely distributed in the lip volume as the boutons of individual PNs are themselves widely scattered across the lip volume (Müller et al., 2002).

MGs are discrete synaptic units and our results strongly suggest that synaptic rearrangements, possibly including the growth of new synapses (Gogolla et al., 2007; Hongpaisan and Alkon, 2007), take place in the MBs during LTM consolidation. Such stable structural changes may be part of a memory trace, but whether they are actually required for memory storage remains to be determined. The MB lips are well suited for memory storage: they are in adequate position for detecting coincident input from olfactory (through the PNs) and reinforcement (through the VUMmx1 neuron; Hammer, 1993) pathways (Menzel, 2001), and may display reverberant activity through feedback loops (Grünewald, 1999; Krashes et al., 2007), in a manner similar to that of the hippocampus (Rolls and Kesner, 2006). Likewise, the presence of many postsynaptic f-actin-rich spines in the MBs (Frambach et al., 2004) provide a suitable substrate for structural synaptic plasticity like in the vertebrate hippocampus (Halpain, 2000; Bramham, 2008). Spines indeed respond with rapid structural reorganization to adapt to presynaptic input changes (Brunig et al., 2004; Lin et al., 2005). Thus, increased activity after conditioning (see above) might trigger spinogenesis and newly formed spines would in turn connect to $\mathrm{PN}$ axons and trigger the formation of additional MG according to the "filopodial model" (Bonhoeffer and Yuste, 2002; Yuste and Bonhoeffer, 2004). Future experiments coupling functional imaging and structural measures of plasticity will directly address this question.

\section{References}

Bitterman ME, Menzel R, Fietz A, Schäfer S (1983) Classical conditioning of proboscis extension in honeybees (Apis mellifera). J Comp Psychol 97:107-119.

Bonhoeffer T, Yuste R (2002) Spine motility. Phenomenology, mechanisms, and function. Neuron 35:1019-1027.

Bramham CR (2008) Local protein synthesis, actin dynamics, and LTP consolidation. Curr Opin Neurobiol 18:524-531.

Brunig I, Kaech S, Brinkhaus H, Oertner TG, Matus A (2004) Influx of extracellular calcium regulates actin-dependent morphological plasticity in dendritic spines. Neuropharmacol 47:669-676. 
Davis RL (2005) Olfactory memory formation in Drosophila: from molecular to systems neuroscience. Annu Rev Neurosci 28:275-302.

Devaud JM, Blunk A, Podufall J, Giurfa M, Grünewald B (2007) Using local anaesthetics to block neuronal activity and map specific learning tasks to the mushroom bodies of an insect brain. Eur J Neurosci 26:3193-3206.

Durst C, Eichmuller S, Menzel R (1994) Development and experience lead to increased volume of subcompartments of the honeybee mushroom body. Behav Neural Biol 62:259-263.

Erber J, Mashur TH, Menzel R (1980) Localization of short-term memory in the brain of the bee, Apis mellifera. Physiol Entomol 5:343-358.

Faber T, Menzel R (2001) Visualizing mushroom body response to a conditioned odor in honeybees. Naturwissenschaften 88:472-476.

Faber T, Joerges J, Menzel R (1999) Associative learning modifies neural representations of odors in the insect brain. Nat Neurosci 2:74-78.

Farris SM, Robinson GE, Fahrbach SE (2001) Experience- and age-related outgrowth of intrinsic neurons in the mushroom bodies of the adult worker honeybee. J Neurosci 21:6395-6404.

Fernandez PC, Locatelli FF, Person-Rennell N, Deleo G, Smith BH (2009) Associative conditioning tunes transient dynamics of early olfactory processing. J Neurosci 29:10191-10202.

Frambach I, Rossler W, Winkler M, Schürmann FW (2004) F-actin at identified synapses in the mushroom body neuropil of the insect brain. J Comp Neurol 475:303-314.

Giurfa M (2007) Behavioral and neural analysis of associative learning in the honeybee: a taste from the magic well. J Comp Physiol A Neuroethol Sens Neural Behav Physiol 193:801-824.

Gogolla N, Galimberti I, Caroni P (2007) Structural plasticity of axon terminals in the adult. Curr Opin Neurobiol 17:516-524.

Groh C, Tautz J, Rössler W (2004) Synaptic organization in the adult honey bee brain is influenced by brood-temperature control during pupal development. Proc Natl Acad Sci U S A 101:4268-4273.

Groh C, Ahrens D, Rossler W (2006) Environment- and age-dependent plasticity of synaptic complexes in the mushroom bodies of honeybee queens. Brain Behav Evol 68:1-14.

Gronenberg W (2001) Subdivisions of hymenopteran mushroom body calyces by their afferent supply. J Comp Neurol 435:474-489.

Gronenberg W, Heeren S, Hölldobler B (1996) Age-dependent and taskrelated morphological changes in the brain and the mushroom bodies of the ant Camponotus floridanus. J Exp Biol 199:2011-2019.

Grünewald B (1999) Morphology of feedback neurons in the mushroom body of the honeybee, Apis mellifera. J Comp Neurol 404:114-126.

Halpain S (2000) Actin and the agile spine: how and why do dendritic spines dance? Trends Neurosci 23:141-146.

Hammer M (1993) An identified neuron mediates the unconditioned stimulus in associative olfactory learning in honeybees. Nature 366:59-63.

Hourcade B, Perisse E, Devaud JM, Sandoz JC (2009) Long-term memory shapes the primary olfactory center of an insect brain. Learn Mem 16:607-615.

Hongpaisan J, Alkon DL (2007) A structural basis for enhancement of longterm associative memory in single dendritic spines regulated by PKC. Proc Natl Acad Sci U S A 104:19571-19576.
Jones TA, Donlan NA, O'Donnell S (2009) Growth and pruning of mushroom body Kenyon cell dendrites during worker behavioral development in the paper wasp, Polybia aequatorialis (Hymenoptera: Vespidae). Neurobiol Learn Mem 92:485-495.

Krashes MJ, Keene AC, Leung B, Armstrong JD, Waddell S (2007) Sequential use of mushroom body neuron subsets during Drosophila odor memory processing. Neuron 53:103-115.

Krofczik S, Khojasteh U, de Ibarra NH, Menzel R (2008) Adaptation of microglomerular complexes in the honeybee mushroom body lip to manipulations of behavioral maturation and sensory experience. Dev Neurobiol 68:1007-1017.

Lin B, Kramár EA, Bi X, Brucher FA, Gall CM, Lynch G (2005) Theta stimulation polymerizes actin in dendritic spines of hippocampus. J Neurosci 25:2062-2069.

Locatelli F, Bundrock G, Müller U (2005) Focal and temporal release of glutamate in the mushroom bodies improves olfactory memory in Apis mellifera. J Neurosci 25:11614-11618.

Maleszka J, Barron AB, Helliwell PG, Maleszka R (2009) Effect of age, behaviour and social environment on honey bee brain plasticity. J Comp Physiol A Neuroethol Sens Neural Behav Physiol 195:733-740.

Menzel R (2001) Searching for a memory trace in a mini-brain, the honeybee. Learn Mem 8:53-62.

Muenz TS, Oberwallner B, Gehring K, Rössler W (2008) Plasticity of synaptic complexes in the mushroom bodies of the honeybee brain depends on age, experience and season. FENS Abstr 4:143.

Müller D, Abel R, Brandt R, Zockler M, Menzel R (2002) Differential parallel processing of olfactory information in the honeybee, Apis mellifera $\mathrm{L}$. J Comp Physiol A Neuroethol Sens Neurol Behav Physiol 188:359-370.

O'Donnell S, Donlan NA, Jones TA (2004) Mushroom body structural change is associated with division of labor in eusocial wasp workers ( $P$ olybia aequatorialis, Hymenoptera: Vespidae). Neurosci Lett 356:159-162.

Rolls ET, Kesner RP (2006) A computational theory of hippocampal function, and empirical tests of the theory. Prog Neurobiol 79:1-48.

Sandoz JC, Galizia CG, Menzel R (2003) Side-specific olfactory conditioning leads to more specific odor representation between sides but not within sides in the honeybee antennal lobes. Neuroscience 120:11371148.

Schwärzel M, Müller U (2006) Dynamic memory networks: dissecting molecular mechanisms underlying associative memory in the temporal domain. Cell Mol Life Sci 63:989-998.

Seid MA, Wehner R (2009) Delayed axonal pruning in the ant brain: a study of developmental trajectories. Dev Neurobiol 69:350-364.

Winston ML (1987) The biology of the honey bee. Cambridge, MA: Harvard UP.

Withers GS, Fahrbach SE, Robinson GE (1993) Selective neuroanatomical plasticity and division of labour in the honeybee. Nature 364:238-240.

Yu D, Ponomarev A, Davis RL (2004) Altered representation of the spatial code for odors after olfactory classical conditioning; memory trace formation by synaptic recruitment. Neuron 42:437-449.

Yuste R, Bonhoeffer T (2004) Genesis of dendritic spines: insights from ultrastructural and imaging studies. Nat Rev Neurosci 5:24-34. 